\title{
Function Indicator Light Device
}

National Cancer Institute

\section{Source}

National Cancer Institute. Function Indicator Light Device. NCI Thesaurus. Code C50284.

A lighted component that is part of a function indicator. 